\title{
EFICIÊNCIA E SELETIVIDADE DE INSETICIDAS NO CONTROLE DO PSILÍDEO (Triozoida sp.) EM GOIABEIRA
}

\author{
FLÁVIA RABELO BARBOSA* \\ EDUARDO ALVES DE SOUZA* \\ KATIA MARIA MEDEIROS DE SIQUEIRA*** \\ WELLINGTON ANTONIO MOREIRA* \\ JOSÉ ADALBERTO DE ALENCAR ${ }^{* * * * *}$ \\ FRANCISCA NEMAURA PEDEROSA HAJI*
}

\begin{abstract}
Verificou-se a eficiência e a seletividade de inseticidas no controle do psilídeo (Triozoida sp.) em goiabeira. Usou-se o delineamento de blocos ao acaso com seis tratamentos e quatro repetições, sendo a unidade experimental constituída por vinte plantas da cultivar Paluma. Os tratamentos, concentrações, formulações e dosagens dos inseticidas (em $\mathrm{mL}$ de produto comercial) foram: 1) Imidacloprid 200 $\mathrm{SL}, 5 \mathrm{~mL} /$ planta; 2) Imidacloprid $100 \mathrm{AL}, 10 \mathrm{~mL} /$ planta; 3) Imidacloprid $200 \mathrm{SC}, 1000 \mathrm{~mL} / \mathrm{ha}$; 4) Betacyflutrin $50 \mathrm{CE}$, $200 \mathrm{~mL} / \mathrm{ha}$; 5) Thiacloprid $480 \mathrm{SC}, 200 \mathrm{~mL} / \mathrm{ha}$ e 6) testemunha. Realizou-se aplicação via tronco do Imidacloprid $200 \mathrm{SL}$ e $100 \mathrm{AL}$, enquanto o Imidacloprid $200 \mathrm{SC}$ e os outros produtos foram pulverizados seis vezes, na folhagem, em intervalos semanais. A eficiência dos produtos foi calculada pela fórmula de Abbott. $\mathrm{O}$ número médio de ramos danificados por psilídeos na testemunha foi 14,24 , diferindo significativamente $(p<0,005 \%)$ das plantas tratadas com Imidacloprid 200 SC $(1,67)$, Thiacloprid 480 SC $(3,37)$, Betacyflutrin 50 CE $(3,72)$, Imidacloprid 100 AL $(6,70)$ e Imidacloprid $200 \mathrm{SL}(9,33)$. Constatou-se a presença dos inimigos naturais Cycloneda sanguinea e Scymnus sp. e de espécimens de aracnídeos, crisopídeos, sirfídeos e tacnídeos. Foram encontrados 5,58 predadores na testemunha e 5,$16 ; 4,44 ; 4,17 ; 2,09$ e 3,94 nos tratamentos $1,2,3,4$ e 5 , respectivamente, o que corresponde às notas $1,1,2,4$ e 2 na escala de seletividade (1 = 0-20\%; 2 = 21-40\%; $3=41-60 \% ; 4=61-100 \%$ de redução populacional de inimigos naturais).
\end{abstract}

PALAVRAS-CHAVE: INSETICIDAS; PSILÍDEO; Triozoida sp.; PRAGAS.

* Engenheiros Agrônomos, Doutores, Embrapa Semi-Árido, Petrolina, PE. (e-mail: flavia@cpatsa.embrapa.br).

** Estagiário Embrapa Semi-Árido, Petrolina, PE.

*** Engenheira Agrônoma, M. Sc., Universidade do Estado da Bahia, Departamento de Tecnologia e Ciências Sociais, Juazeiro, BA.

**** Engenheiro Agrônomo, M. Sc., Embrapa Semi-Árido. 


\section{INTRODUÇÃO}

Durante seu desenvolvimento, a goiabeira é atacada por diversos insetos-praga que provocam diferentes tipos de danos. MARICONE \& SOUBIHE SOBRINHO (1961) registraram no Brasil mais de cem espécies de insetos em goiabeira. NAKANO \& SILVEIRA NETO (1968) relataram a presença de Triozoidasp. (Hemiptera, Psyllidae) em goiabeira, em diversas regiões do Estado de São Paulo. No Submédio São Francisco, o psilídeo Triozoida sp. é a principal praga (BARBOSA et al., 1999; SILVA, 2000).

Os psilídeos são insetos sugadores de seiva. O macho apresenta coloração esverdeada com a face dorsal do tórax e do abdome de coloração preta, medindo, em média, $2,0 \mathrm{~mm}$ de comprimento. A fêmea é verde amarelada com, aproximadamente, 2,4 $\mathrm{mm}$ de comprimento. As ninfas, de formato achatado e coloração rósea, apresentam-se recobertas por excreção de cera de coloração esbranquiçada e ao sugarem a seiva nas bordas das folhas injetam toxinas. As folhas atacadas tornam-se enroladas e deformadas, de coloração amarelada ou avermelhada, apresentando, posteriormente, aspecto necrosado. Examinando-se o interior das partes enroladas das folhas são observadas colônias de ninfas (GALLO et al., 1988; PEREIRA \& BORTOLI, 1998).

No Submédio São Francisco, a poda é praticada durante o ano todo, facilitando o ataque dos psilídeos, pois as brotações são preferidas por estes insetos. Embora o controle químico desta praga seja realizado, rotineiramente, na cultura da goiabeira não há produtos registrados no mercado para esta frutífera. Em 1997, aproximadamente, foi registrado no Brasil o inseticida Imidacloprid para o controle de pragas de citros, produto sistêmico que é aplicado no tronco da planta (WINNER..., 1997). Em experimentos realizados com este inseticida, aplicado via tronco, visando o controle de psilídeos, em citros e goiabeira, constatou-se redução significativa dos danos causados por estes insetos (FLORIM et al., 1998; BARBOSA et al., 1999).

No contexto da filosofia do Programa de Manejo de Pragas, o uso de inseticidas constitui-se numa das táticas disponíveis para o agricultor regular as populações de insetos potencialmente danosos à cultura evitando perdas que possam comprometer a produtividade ou a qualidade do produto. Além de avaliar o efeito dos inseticidas sobre os insetos-praga torna-se importante verificar o impacto destes sobre seus inimigos naturais.

O objetivo deste trabalho foi verificar a eficiência e a seletividade dos inseticidas Imidacloprid, em três formulações e dosagens, do Betacyflutrin e do Thiacloprid no controle do psilídeo Triozoida sp. em goiabeira. 


\section{MATERIAL E MÉTODOS}

O experimento foi conduzido em pomar comercial, no Projeto de Irrigação Senador Nilo Coelho, no município de Petrolina-PE. Os tratamentos, concentrações, formulações e dosagens dos inseticidas (em $\mathrm{mL}$ de produto comercial) foram: 1) Imidacloprid $200 \mathrm{SL}, 5 \mathrm{~mL} /$ planta; 2) Imidacloprid 100 AL, 10 mL/planta; 3) Imidacloprid 200 SC, 1000 mL/ha; 4) Betacyflutrin $50 \mathrm{CE}, 200 \mathrm{~mL}$./ha; 5) Thiacloprid 480 SC, $200 \mathrm{~mL} / \mathrm{ha}$ e 6) testemunha. Foi realizada apenas uma aplicação do Imidacloprid 200 SL e 100 AL, sendo a dosagem subdividida nas hastes primárias da planta, enquanto o Imidacloprid $200 \mathrm{SC}$ e os outros produtos testados foram pulverizados seis vezes, em intervalos semanais. Imidacloprid $200 \mathrm{SL}$ e $100 \mathrm{AL}$ foram aplicados puros, via tronco, por meio de equipamento manual específico, enquanto Imidacloprid 200 SC, Betacyflutrin 50 CE e Thiacloprid 480 SC foram aplicados nas folhas com pulverizador costal manual. O experimento foi iniciado quando observou-se índice de infestação da praga superior a $80 \%$.

Usou-se o delineamento experimental de blocos ao acaso com quatro repetições, sendo a unidade experimental constituída por vinte plantas da cultivar Paluma, com 12 meses de idade, em plena brotação. As amostragens do número de ramos danificados por psilídeos e de inimigos naturais foram realizadas aos 15, 25, 35, 45 e 55 dias após a primeira aplicação dos inseticidas, caminhando-se ao redor da planta e anotando-se o número e tipos de inimigos naturais presentes. Para a análise de variância, os dados referentes ao número de galhos danificados foram transformados em $\sqrt{x+1}$. As médias foram comparadas pelo teste de Tukey, ao nível de $5 \%$ de probabilidade e as porcentagens de eficiência dos produtos (E) calculadas pela fórmula de ABBOTT (1925). As porcentagens de redução populacional dos inimigos naturais foram mensuradas por meio da fórmula de ABBOTT (1925), comparando-se a média do número de inimigos naturais na testemunha com a média do número de inimigos naturais nos tratamentos. Estas foram enquadradas na seguinte escala de seletividade: $1=0-20 \% ; 2=21-40 \% ; 3=41-60 \%$; $4=61-100 \%$ de redução populacional de inimigos naturais (EMBRAPA, 1995).

\section{RESULTADOS E DISCUSSÃO}

Os inseticidas causaram redução significativa no número total de galhos danificados por psilídeos (Tabela 1), sendo constatado na testemunha 14,24 galhos danificados. Não foram observadas diferenças 
significativas entre Imidacloprid $200 \mathrm{SL}$ e Imidacloprid $100 \mathrm{AL}$, assim como entre Imidacloprid $100 \mathrm{AL}$, Betacyflutrin $50 \mathrm{CE}$ e Thiacloprid $100 \mathrm{AL}$ e, ainda, entre Betacyflutrin 50 CE, Thiacloprid 100 AL e Imidacloprid 200 SC.

\section{TABELA 1 - EFEITO DOS TRATAMENTOS NO NÚMERO MÉDIO TOTAL DE GALHOS DANIFICADOS POR PSILÍDEOS E NA POPULAÇÃO DE INIMIGOS NATURAIS, ATÉ 55 DIAS APÓS A PRIMEIRA APLICAÇÃO DOS INSETICIDAS - PETROLINA - PE/1999}

\begin{tabular}{lcccc}
\hline & \multicolumn{4}{c}{ P a r â m e t r o } \\
\cline { 2 - 5 } T r a t a m e n t o & $\begin{array}{c}\text { Galhos } \\
\text { danificados } \\
\left(\mathrm{N}^{\circ}\right)\end{array}$ & $\begin{array}{c}\text { Eficiência }^{2} \\
(\%)\end{array}$ & $\begin{array}{c}\text { Inimigos } \\
\text { naturais } \\
\left(\mathrm{N}^{\circ}\right)\end{array}$ & $\begin{array}{c}\text { Seletividade } \\
(\text { Nota })^{3}\end{array}$ \\
\hline Testemunha & $14,24 \mathrm{a}$ & - & 5,58 & - \\
Imidacloprid 200 SL & $9,33 \mathrm{~b}$ & 37,96 & 5,16 & 1 \\
Imidacloprid 100 AL & $6,70 \mathrm{bc}$ & 55,12 & 4,44 & 1 \\
Betacyflutrin 50 CE & $3,72 \mathrm{~cd}$ & 72,26 & 2,09 & 4 \\
Thiacloprid 480 SC & $3,37 \mathrm{~cd}$ & 77,59 & 3,94 & 2 \\
Imidacloprid 200 SC & $1,67 \mathrm{~d}$ & 88,90 & 4,17 & 2
\end{tabular}

${ }^{1}$ Médias seguidas pela mesma letra, na coluna, não diferem entre si pelo teste de Tukey ao nível de $5 \%$ de probabilidade (valores não-transformados).

${ }^{2}$ Calculada pela fórmula de ABBOTT (1925).

${ }^{3} 1=0-20 \% ; 2=21-40 \% ; 3=41-60 \% ; 4=61-100 \%$ de redução populacional dos inimigos naturais (Cycloneda sanguinea, Scymnus sp., Aracnídeos, Crisopídeos, Sirfídeos, Tacnídeos).

A percentagem de eficiência média dos produtos testados no controle de psilídeos, até 55 dias após a primeira aplicação dos inseticidas, foi de 37,96\% para Imidacloprid 200 SL, 55,12\% para Imidacloprid 100 AL, 88,90\% para Imidacloprid 200 SC, 72,26\% para Betacyflutrin e $77,59 \%$ para Thiacloprid $100 \mathrm{AL}$ (Tabela 1). BARBOSA et al. (1999) constataram 
eficiência de $81,5 \%$ no controle do psilídeo ( Triozoida sp.) em goiabeira, 45 dias após a aplicação (via tronco) de Imidacloprid. Do mesmo modo, FLORIM et al. (1998) observaram alta eficiência de Imidacloprid 200 SC, no controle do psilídeo (Diaphorina citri), em citros, até 60 dias após a aplicação do produto.

A eficiência dos inseticidas aplicados no tronco (Imidacloprid 200 SL e Imidacloprid $100 \mathrm{AL}$ ) no controle do psilídeo foi crescente dos 15 aos 55 dias da aplicação. Para o Imidacloprid 200 SL, a eficiência neste período variou de 6,79 a $100 \%$, enquanto para o Imidacloprid $100 \mathrm{AL}$ variou de 22,72 a $100 \%$ (Tabela 2). A baixa eficiência inicial destes produtos resultou em baixa eficiência média no período de 15 a 55 dias (Tabela 1).

Com relação aos inimigos naturais constatou-se a presença dos coccinelídeos Cycloneda sanguinea e Scymnus sp. e de espécimens de aracnídeos, crisopídeos, sirfídeos e tacnídeos. O número médio de inimigos naturais na testemunha foi de 5,58 , enquanto nas plantas tratadas com Imidacloprid 200 SL, Imidacloprid 100 AL, Imidacloprid 200 SC, Betacyflutrin 50 CE e Thiacloprid 480 SC foi de 5,$16 ; 4,44 ; 4,17 ; 2,09$ e de 3,94 , respectivamente. Tais resultados corresponderam, respectivamente, às notas 1, 1, 2, 4 e 2 na escala de seletividade. O impacto na população dos insetos benéficos foi menor quando os inseticidas foram aplicados no tronco, comparativamente aos produtos pulverizados (Tabela 1). 0 número médio de inimigos naturais bem como seus tipos, nos diferentes tratamentos, estão relacionados na Tabela 3.

\section{CONCLUSÃO}

A aplicação de Imidacloprid $200 \mathrm{SL}$, Imidacloprid $100 \mathrm{AL}$, Imidacloprid 200 SC, Betacyflutrin 50 CE e Thiacloprid 480 SC reduziu, significativamente, os danos do psilídeo Triozoida sp. em goiabeira.

Os coccinelídeos Cycloneda sanguinea e Scymnus sp. e espécimens de aracnídeos, crisopídeos, sirfídeos e tacnídeos estão associados, agindo como inimigos naturais do psilídeo em pomares de goiabeira no Vale do Submédio São Francisco.

Os inseticidas Imidacloprid $200 \mathrm{SL}$ e Imidacloprid $100 \mathrm{AL}$ apresentaram maior seletividade aos inimigos naturais que Imidacloprid 200 SC, Betacyflutrin 50 CE e Thiacloprid 480 SC.

A eficiência do Imidacloprid 200 SL e do Imidacloprid 100 AL no controle do psilídeo Triozoida sp. em goiabeira não foi observada logo após a sua aplicação, sendo crescente a partir dos 15 dias. 

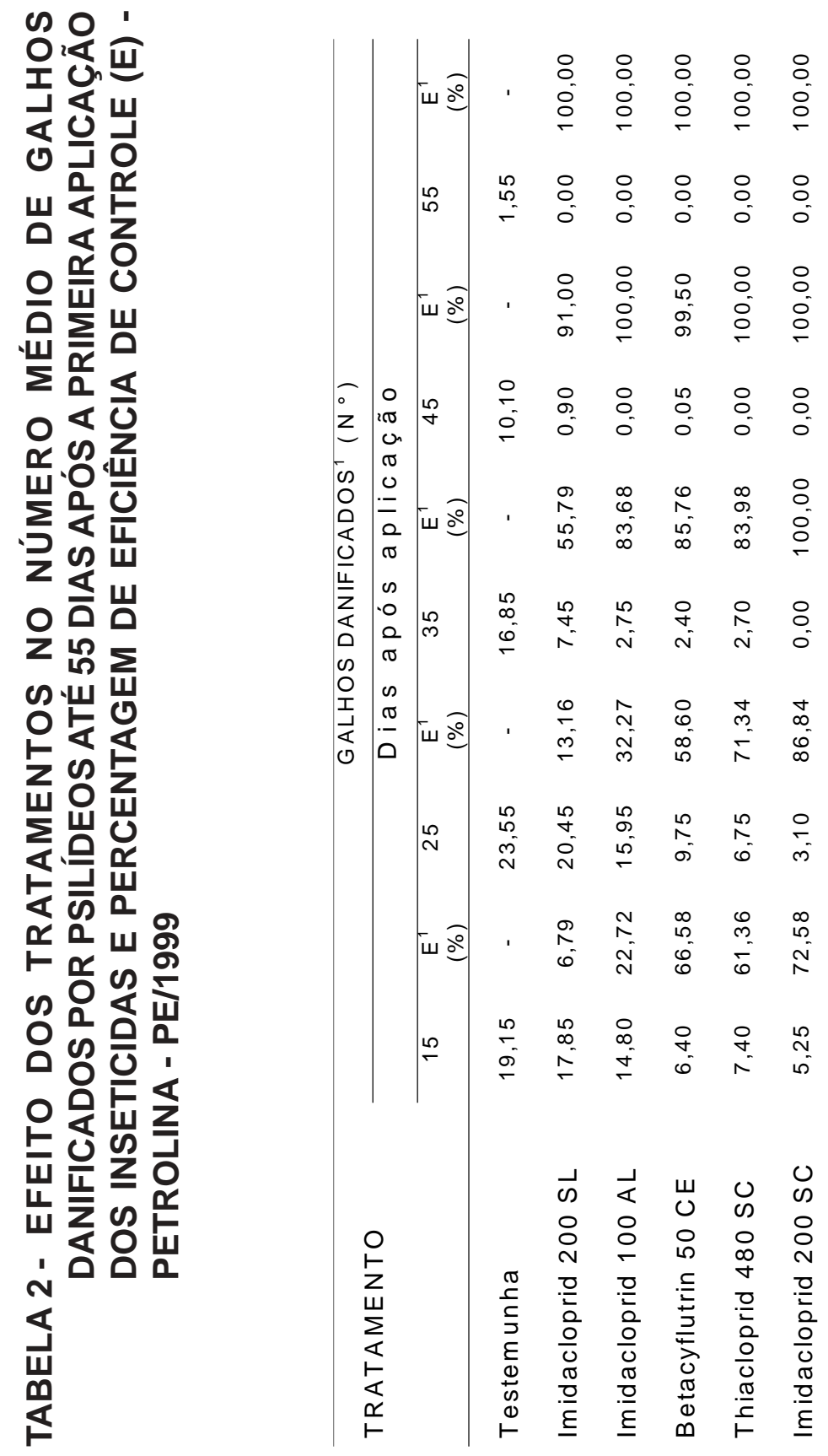

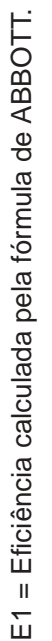




\section{TABELA 3 - EFEITO DOS TRATAMENTOS NO NÚMERO MÉDIO DE INIMIGOS NATURAIS ATÉ 55 DIAS APÓS A PRIMEIRA APLICAÇÃO DOS INSETICIDAS - PETROLINA - PE/1999}

\begin{tabular}{lccccccc}
\hline TRATAMENTO & \multicolumn{7}{c}{ INIMIGOS NATURAIS (N ${ }^{\circ}$} \\
\cline { 2 - 8 } & Aranha & Crisopídeo & Cycloneda & Scymnus & Sirfídeo & Tacnídeo & TOTAL \\
\hline Testemunha & 1,34 & 1,07 & 0,10 & 1,40 & 1,64 & 0,03 & 5,58 \\
Imidacloprid 200 SL & 2,00 & 0,96 & 0,06 & 0,99 & 1,11 & 0,04 & 5,16 \\
Imidacloprid 100 AL & 1,97 & 0,99 & 0,02 & 0,59 & 0,87 & 0,00 & 4,44 \\
Betacyflutrin 50 CE & 1,03 & 0,22 & 0,02 & 0,47 & 0,34 & 0,01 & 2,09 \\
Thiaclopirid 480 SC & 1,87 & 0,76 & 0,00 & 0,51 & 0,80 & 0,00 & 3,94 \\
Imidacloprid 200 SC & 1,71 & 0,78 & 0,07 & 0,50 & 1,10 & 0,01 & 4,17 \\
& & & & & & & \\
\hline
\end{tabular}

\section{Abstract \\ EFFICIENCY AND SELECTIVITY OF INSECTICIDES IN THE CONTROL OF Triozoida sp. IN GUAVA PLANTS}

The efficiency and selectivity of insecticides in the control of Triozoida sp. (Hemiptera, Psylliidae) in guava plants were evaluated. The field experiment consisted of a randomized complete block design with six treatments and four replications, each one formed by twenty plants of guava cv. Paluma. The treatments, concentrations, formulations and levels, (comercial product $/ \mathrm{mL}$ ) were: 1 . Imidacloprid $200 \mathrm{SL}, 5 \mathrm{~mL}$ c.p./plant; 2. Imidacloprid 100 AL, 10 mL c.p./plant; 3. Imidacloprid 200 SC, 1000 mL c.p./ha; 4. Betacyflutrin 50 CE, $200 \mathrm{~mL}$ c.p./ha; 5 . Thiacloprid 480 SC, $200 \mathrm{~mL}$ c.p./ha, and 6. control (without insecticide). Imidacloprid $200 \mathrm{SL}$ and $100 \mathrm{AL}$ were applied via trunk only once, whereas Imidacloprid 200 SC and the other insecticides were sprayed on the canopy six times at 7 day interval. The average efficiency was calculated by Abbot formula. The average number of damaged branches for the control was 14.24 , significantly different $(p<0,005 \%)$ from plants treated with Imidacloprid 200 SC (1.67), Thiacloprid 480 SC (3.37), Betacyflutrin 50 CE (3.72), Imidacloprid 100 AL (6.70) and Imidacloprid 200 SL (9.33). The occurrence of natural enemies, such as, Cycloneda sanguinea, Scymnus spp., arachnids, crisopids, sirfids, and stafilinids was detected. It was found 5.58 in the control, and 5.16; 4.44; 4.17; 2.09 and 3.94, respectively, in the treatments 1, 2, 3, 4 e 5, corresponding, respectively, in the selective scale, to grades $1,1,2$, 4 e $2(1=0-20 \% ; 2=21-40 \% ; 3=$ $41-60 \% ; 4=61-100 \%$ of populacional reduction of natural enemies).

KEY-WORDS: INSECTICIDES; Triozoida sp.; PLAGUES. 


\section{REFERÊNCIAS}

1 ABBOTT, W.S. A method of computing the effectiveness of an inseticide. Journal Economic Entomology, v. 18, n. 1, p. 265-267, 1925.

2 BARBOSA, F.R.; SANTOS, A.P. DOS; HAJI, A.T.; MOREIRA, W.A.; HAJI, F.NP.; ALENCAR, J.A. Eficiência e seletividade do Imidacloprid e lambdacyhalothrin no controle do psilídeo (Triozoida sp.), em goiabeira. Revista Brasileira de Fruticultura, v. 21, n. 3, p. 385387, 1999.

3 EMBRAPA. Centro Nacional de Pesquisa de Soja. Recomendações técnicas para a cultura da soja na região Central do Brasil 1995/96. Londrina: EMBRAPA, CNPSo, 1995. 149 p. (Documentos, 88).

4 FLORIM, A.C.P.; NAKANO, O.; SALVO, S. Eficiência do Imidacloprid no controle do psilídeo (Diaphorina citro.) aplicado via tronco. In: CONGRESSO BRASILEIRO DE ENTOMOLOGIA, 17., Rio de Janeiro, 1998. Resumos... Seropédica: Universidade Federal Rural do Rio de Janeiro, 1998. p. 159.

5 GALLO, D.; NAKANO, O; SILVEIRA NETO, S.; CARVALHO, R.P.L.; BATISTA, G.C.; BERTI FILHO, E.; PARRA, J.R.P.; ZUCCHI, R.A.; ALVES, S.B.; VENDRAMIN, J.D. Manual de entomologia agrícola. São Paulo: Agronômica Ceres, 1988. 649 p.

6 MARICONE, F.A.M.; SOUBIHE SOBRINHO, J. Contribuição para o conhecimento de alguns insetos que depredam a goiabeira (Psidium guajava L.). Piracicaba: ESALQ, Instituto de Genética, 1961. $57 \mathrm{p}$.

7 NAKANO, O.; SILVEIRA NETO, S. Contribuição ao estudo de Triozoida sp. Crawf., praga da goiabeira. Ciência e Cultura, v.20, n. 2, p.263-264, 1968.

8 PEREIRA, F.M.; BORTOLI, S. A. de. Pragas da goiabeira. In: SOBRINHO, R.B.; CARDOSO, J.E.; FREIRE, F. das C. (Eds.). Pragas de fruteiras tropicais de importância agroindustrial. Brasília: Embrapa, SPI, 1998. p.119-130.

9 SILVA, D.A.M. da. Goiabeira (Psidium guajava): cultivo sob condição irrigada. 2. ed. Recife: SEBRAE-PE, 2000. 40 p. (SEBRAEPE, Agricultura, 6).

10 WINNER: inovação no controle de pragas em citros. Correio Agrícola, São Paulo, n.2, p.6, 1997. 